\title{
A comprehensive comparison of computational methods on propeller modelling of an AUV
}

\author{
Guo Hao Ang \\ National Centre for Maritime \\ Engineering and Hydrodynamics \\ Australian Maritime College \\ University of Tasmania \\ Launceston, Australia \\ guohao.ang@utas.edu.au
}

\author{
Shuangshuang Fan \\ National Centre for Maritime \\ Engineering and Hydrodynamics \\ Australian Maritime College \\ University of Tasmania \\ Launceston, Australia \\ shuangshuang.fan@utas.edu.au
}

\author{
Yuting Jin \\ National Centre for Maritime \\ Engineering and Hydrodynamics \\ Australian Maritime College \\ University of Tasmania \\ Launcestion, Australia \\ yuting.jin@utas.edu.au
}

\author{
HuiSheng Lim \\ National Centre for Maritime \\ Engineering and Hydrodynamics \\ Australian Maritime College \\ University of Tasmania \\ Launcestion, Australia \\ hui.lim@utas.edu.au
}

\author{
Christopher K. H. Chin \\ National Centre for Maritime Engineering \\ and Hydrodynamics \\ Australian Maritime College \\ University of Tasmania \\ Launceston, Australia \\ c.chin@utas.edu.au
}

\author{
Shuhong Chai \\ National Centre for Maritime Engineering \\ and Hydrodynamics \\ Australian Maritime College \\ University of Tasmania \\ Launceston, Australia \\ shuhong.chai@utas.edu.au
}

Neil Bose

Bruneau Centre for Research and Innovation Memorial University of Newfoundland

St. John's, NL, Canada

nbose@mun.ca

\begin{abstract}
The propeller of a self-propelled marine vehicle is the key to understanding the hull-propeller interactions. The presence of a propeller not only provides the necessary propulsion force, but also significantly modifies the flow field around the marine vehicle. This modification made to flow field influences the hydrodynamic loading experienced by the marine vehicle and thus has an impact on its maneuvering characteristics. There are various methods to represent the actions of a propeller for a range of marine applications within CFD. Actuator disc or virtual disc is one of the earliest and most commonly used approach. This approach solves the body force generated as an equally distributed force over a cylindrical disc while ignoring the geometry of the propeller. Another method to evaluate the force of a propeller is direct simulations, which replicate a propeller based on its true geometry within CFD. This method utilizes the overset mesh technology within CFD that enables the propeller to rotate independently with respect to the background mesh. The present paper primarily used commercially available CFD software package, Star-CCM+ to investigate the accuracy and feasibility of representing the propeller of an Explorer Class AUV using the earlier mentioned methods. The solved advance ratio, and forward velocity from the two methods was benchmarked against openwater trial data.
\end{abstract}

Keywords-CFD, AUV, propeller modelling, self-propulsion, virtual disc

\section{INTRODUCTION}

The propeller of a self-propelled marine vehicle is the key to understanding the hull-propeller interactions. The presence of a propeller not only provides the propulsion force but also significantly modifies the flow field around the marine vehicle. This modification made to the flow field influences the hydrodynamic loading experienced by the marine vehicle and in return influence the vehicle's manoeuvring characteristics [1]. The effects of a propeller on a marine vehicle are commonly studied through either experimental fluid dynamics
(EFD) or computational fluid dynamics (CFD). With the increasing computational power and availability, more researchers have turned to CFD due to its ability to offer a quick solution with high accuracy for its predictions of flow field around a body in fluid and the manoeuvring characteristics of a marine vehicle. Furthermore, CFD methods are more versatile and convenient as compared to EFD.

There are various methods to represent the actions of a propeller for a range of marine applications within CFD, such as actuator disc or direct simulation. Actuator disc or virtual disc method is one of the earliest and most commonly used approach since its introduction in 1865 by Rankine [2]. This approach solves the body force generated as an equally distributed force over a cylindrical disc while ignoring the geometry of the propeller, and due to its computational efficiency, it has often been used for the study of any propeller related marine applications. A recent study by [3] compared the modified Hough and Ordway model against the blade element theory and its respective effects on free turning manoeuvring characteristics for a surface vessel. The mentioned study highlighted a few of the many different models for an actuator disc, and the percentage error between these models against the experimental data are less than $10 \%$. Studies by [4] and [5] have illustrated the robustness of actuator disc in terms of time and cost when applied to the studying of self-propulsion in marine applications.

Another method to evaluate the force of a propeller is direct simulations, which replicate a propeller based on its true geometry within CFD. This method utilizes the overset mesh function within a CFD software package, which enables the propeller to rotate independently with respect to the background mesh. With increasing computational power, this method can solve geometry and flow that are more complicated than before. This approach was used by [6] to perform a series of freerunning simulation for the Postdam Model Basin Korean 
container ship (KCS) model with independent moving rudder and rotating propeller. The study showed that despite the long computational time, direct simulation can predict the propeller's characteristics and the ship's motion. Furthermore, the direct simulation approach can also provide a more realistic effect of the hull-propeller interactions as illustrated by [7] who investigated the hull-propeller interactions for a submarine at near-surface conditions. In the paper, it was mentioned that the interactions between hull-propeller plays an important role in power performance prediction and the inclusion of a discretized propeller would provide a more realistic geometry of the submarine. In the comparison study by [8] between the direct overset simulation and coupled CFD with potential flow solver for the evaluation of submarine's manoeuvres, it was pointed out that the coupled approach yields less details in the flow field and has higher error.

The above-mentioned articles are some of the many works carried out previously that hopes to provide an accurate representation of a propeller within CFD for the investigation of hull-propeller interaction for a marine vehicle. However, to date, there are limited comparison works carried out for AUVs regarding similar problems within CFD as AUVs behave differently as compared to a surface vessel or a submarine. To this end, this paper aims to provide a comprehensive comparison between two different modelling for an AUV within CFD i.e. virtual disc and direct simulation that may be used for the studying of hull-propeller interactions.

The present study was primarily conducted using the commercially available CFD software package, Star-CCM+. The comparison study investigated the accuracy and feasibility of representing the propeller of an Explorer Class AUV using either the actuator disc method or direct simulation method. Thus, to compare the performance of these methods, free running manoeuvring (i.e. straight-line runs, zig-zag manoeuvrings and turning circles manoeuvrings) were performed and the hydrodynamic coefficients and propulsion characteristics obtained from each run were compared and benchmarked against open-water trial conducted by the International Submarine Engineering (ISE). The open-water curve of the Explorer Class AUV's propeller was first predicted through CFD method as its open-water curve was not available in the public domain. The predicted open-water curve data was used to program the virtual disc. In this paper, the comparison of the different propeller representations was based on the advance ratio, and forward velocity, which were obtained from the simulations and validated against the open-water trial. This paper will present the cases when the AUV is travelling in a straight-line. However, hydroplanes were not modelled in all simulations.

\section{AUV GEOMETRY}

The AMC Explorer Class AUV was used throughout this study. The overall configuration of the Explorer AUV is rather unique compared to common AUVs. As illustrated in Fig. 2, the AUV has a total of 6 hydroplanes, 2 fore hydroplanes and 4 aft hydroplanes arranged in an X-form and propelled by a twinbladed Wageningen B-Series propeller. The Explorer AUV's and its propeller's principle particulars are listed in Table I and Table II respectively. Although the Wageningen B-series propeller is a commonly used propeller in the marine industry, the twin-bladed B-Series propeller is used for the Explorer that has an area ratio of 0.1 , since there are no publicly available open-water curve data for this propeller. Hence, its open-water curve must be predicted.

\section{COMPUTATIONAL METHODS}

\section{A. RANS Equations}

To evaluate the flow around the vehicle, a model based on RANS equations was used. Assuming the fluid is incompressible, the mean flow field governed by the RANS equations can be expressed as [9]:

$$
\begin{gathered}
\partial U_{j} / \partial x_{j}=0 \\
\frac{\partial U_{i}}{\partial t}+\frac{\partial}{\partial x_{j}}\left(U_{i} U_{j}\right)=-\frac{1}{\rho} \frac{\partial P}{\partial x_{i}}+\frac{1}{\rho} \frac{\partial}{\partial x_{j}}\left(2 \mu S_{i j}-\rho \bar{u}_{i}^{\prime} \bar{u}_{j}^{\prime}\right) \\
S_{i j}=\frac{1}{2}\left(\frac{\partial U_{i}}{\partial x_{j}}+\frac{\partial U_{j}}{\partial x_{i}}\right)
\end{gathered}
$$

Where $x$ and $t$ represent space and time, while $i$ and $j$ represent the spatial indexes. $\rho$ and $\mu$ represent density and viscosity respectively. $U_{i(j)}$ represent the time-averaged velocity and $P$ is the pressure field. The term $S_{i j}$ refer to the mean strain-rate tensor. The term $\bar{u}_{i}^{\prime} \bar{u}_{j}^{\prime}$ represent the symmetrical Reynolds stress tensor which consist of six components.

\section{B. 6 DOF and coordinate system}

A 6 Degree of Freedom (DOF) motion module was implemented into Star-CCM+ for the AUV hydrodynamics applications. Two set of coordinate systems were used, namely earth system $\left(o^{\prime}, x^{\prime}, y^{\prime}, z^{\prime}\right)$ and AUV system $(o, x, y, z)$ as illustrated in Fig. 1.

As illustrated in Fig. 1 for the AUV system, the longitudinal $x$-axis points aft to fore, the transversal $y$-axis points from port to starboard, and the vertical $z$-axis points upwards.

\section{VERIFICATION AND VALIDATION}

Verification is the process of assessing the proposed CFD model's numerical uncertainty. Validation is the process of assessing the numerical uncertainty of the proposed CFD model by benchmarking the numerical solution against real data or experimental data. The validation and verification procedure for this study are based on the procedure outlined by [10] with minor simplifications.

The validation and verification studies were conducted for a five-bladed propeller. The study was not carried out for the Explorer's twin bladed propeller, as there was no public available open-water curve. Although, both propellers are different, the methodology to obtain both open-water curves are the same. Therefore, if the methodology to predict the fivebladed propeller is accurate, this would indicate that the same methodology can be used to predict the open-water curve for the twin-bladed propeller. 


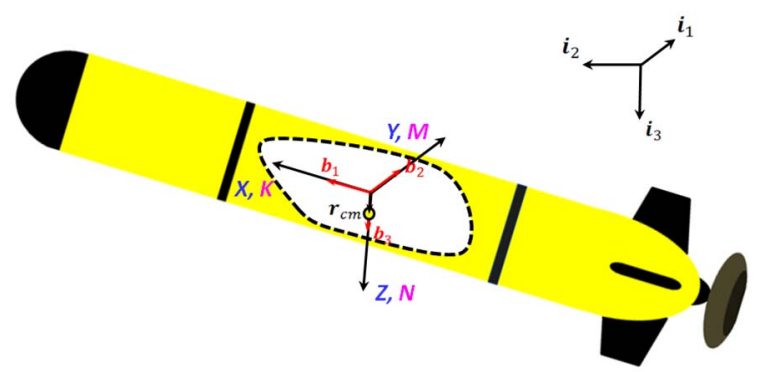

Fig. 1. Reference frame of the AUV in study

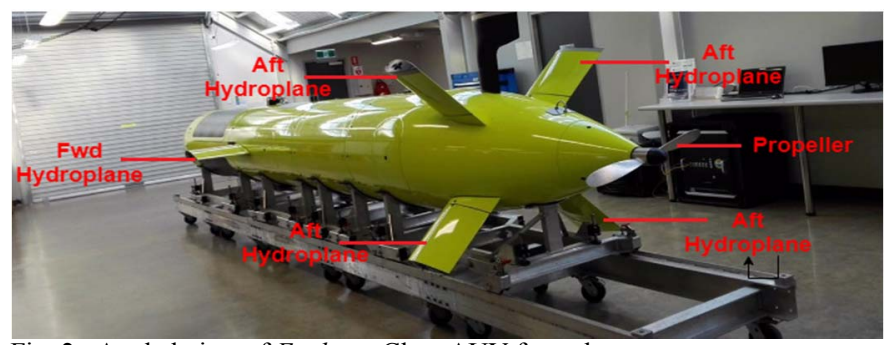

Fig. 2. Angled view of Explorer Class AUV from the stern

TABLE I. Principle particulars of AMC Explorer Class AUV

\begin{tabular}{|c|c|}
\hline Parameters & Full-scale dimension \\
\hline Length $(\mathrm{m})$ & 6.6 \\
\hline Diameter $(\mathrm{m})$ & 0.74 \\
\hline Slender ratio L/D & 8.92 \\
\hline Mass $(\mathrm{kg})$ & 1500 \\
\hline Speed $(\mathrm{m} / \mathrm{s})$ & $1-2.5$ \\
\hline
\end{tabular}

TABLE II. Principle particulars of Wageningen B-Series propeller

\begin{tabular}{|c|c|}
\hline Parameters & Full-scale dimension \\
\hline Diameter $(\mathrm{m})$ & 0.65 \\
\hline Area ratio & 0.1 \\
\hline P/D & 0.87 \\
\hline No. of blades & 2 \\
\hline
\end{tabular}

\section{A. Open-Water Curve for a five-bladed propeller}

Throughout all the runs, the revolution per second (RPS) $n$ was fixed at 13.33RPS while the advance speed was varied accordingly to achieve the desired advance coefficient $J$. The following formulae were used to obtain the thrust coefficient $K_{T}$, torque coefficient $K_{Q}$, and efficiency $\eta_{0}$ for each advance coefficient:

$$
\begin{gathered}
K_{T}=T / \rho n^{2} D^{4} \\
K_{Q}=Q / \rho n^{2} D^{5} \\
\eta_{0}=J K_{T} / 2 \pi K_{Q} \\
J=V_{A} / n D
\end{gathered}
$$

Where $T$ and $Q$ represent thrust and torque respectively, and $V_{A}$ represents the advance velocity. $D$ is the diameter of the propeller.
The properties of the five-bladed propeller are as outlined in [11].

\section{B. Numerical uncertainty analysis}

The numerical uncertainty analysis methodology used in this study was as outlined by [10]. The numerical uncertainty $U_{S N}$ was derived as a combination of grid convergence uncertainty $U_{G}$ and time-step convergence uncertainty $U_{T}$ as illustrated in (8):

$$
U_{S N}=\sqrt{U_{G}^{2}+U_{T}^{2}}
$$

Three distinctive set of results are required for each of the analysis. For grid analysis, three mesh sizes with an increment ratio $r_{i}$ of square root 2 is required. The three mesh sizes can be categorised as fine mesh $h_{1}$, medium mesh $h_{2}$ and coarse mesh $h_{3}$. For the time-step analysis, the time-step increment ratio $r_{i}$ is a fixed value of 2 . The three time-step can be categorised as shortest time-step $h_{1}$, medium time-step $h_{2}$ and longest timestep $h_{3}$.

The convergence ratio $R_{i}$ is then defined as the difference in solution $S$ from the three grids or time-steps as follows:

$$
R_{i}=\left(S_{2}-S_{1}\right) /\left(S_{3}-S_{2}\right)
$$

The three possible convergence conditions are:

1) $0<R_{i}<1$ Monotonic convergence (MC)

2) $R_{i}<0 \quad$ Oscillatory convergence (OC)

3) $R_{i}>1 \quad$ Monotonic divergence (MD)

For the third condition, grid uncertainty cannot be estimated.

The numerical uncertainty analysis for both grid and timestep convergence study was performed for the case of openwater flow for the five-bladed propeller at an advance speed of $0.8 \mathrm{~m} / \mathrm{s}$. The results for the grid uncertainty and time-step uncertainty study are summarized in Table III and Table IV respectively.

Through the grid uncertainty study, both torque coefficient and efficiency achieved monotonic convergence while thrust coefficient achieved oscillatory convergence. The uncertainty of the finest mesh size is less than $1 \%$ and the associating gird's uncertainty are less than $1 \%$ of the finest grid solution. For the time-step uncertainty study, all three variables have achieved monotonic convergence. The uncertainty for the shortest timestep is less than $1 \%$ and the associated time-step uncertainty are less than $1 \%$. Therefore, both uncertainty studies suggest that the applied methodology is capable of yielding solutions with reasonably small and acceptable uncertainty.

\section{Validating against experimental results}

Validation of the simulated results was performed through comparing against the benchmark experimental data from [11]. The validation results are shown in Fig. 3 and Table V. 
TABLE III. Grid spacing uncertainty analysis results

\begin{tabular}{|c|c|c|c|c|c|c|c|c|}
\hline \multirow{2}{*}{ Variables } & \multirow{2}{*}{$\mathbf{r}_{\mathrm{G}}$} & \multicolumn{3}{|c|}{ Solutions } & \multirow{2}{*}{$\mathbf{R}_{\mathrm{G}}$} & \multirow{2}{*}{ Convergence } & \multirow{2}{*}{$\delta_{G}\left(\% S_{1}\right)$} & \multirow{2}{*}{$\mathrm{U}_{\mathrm{G}}\left(\% \mathrm{~S}_{1}\right.$} \\
\hline & & $\mathbf{S}_{1}$ & $\mathbf{S}_{2}$ & $\mathbf{S}_{3}$ & & & & \\
\hline Kt & $\sqrt{2}$ & 0.3174 & 0.3170 & 0.3183 & -0.2740 & $\mathrm{OC}$ & N.A & 0.2016 \\
\hline ETA0 & $\sqrt{2}$ & 0.4039 & 0.3991 & 0.3896 & 0.5116 & $\mathrm{MC}$ & -0.5063 & 0.9882 \\
\hline
\end{tabular}

TABLE IV. Time-step uncertainty analysis results

\begin{tabular}{|c|c|c|c|c|c|c|c|c|}
\hline \multirow[b]{2}{*}{ Variables } & \multirow{2}{*}{$\mathbf{r}_{\mathrm{T}}$} & \multicolumn{3}{|c|}{ Solutions } & \multirow{2}{*}{$\mathbf{R}_{\mathbf{T}}$} & \multirow{2}{*}{ Convergence } & \multirow{2}{*}{$\boldsymbol{\delta}_{\mathrm{T}}\left(\% \mathrm{~S}_{1}\right)$} & \multirow{2}{*}{$\mathrm{U}_{\mathrm{T}}\left(\mathrm{O}_{\mathbf{0}} \mathrm{S}_{\mathbf{1}}\right)$} \\
\hline & & $\mathbf{S}_{1}$ & $\mathbf{S}_{2}$ & $\mathbf{S}_{3}$ & & & & \\
\hline Kt & 2 & 0.3174 & 0.3170 & 0.3183 & 0.6405 & $\mathrm{MC}$ & 0.0502 & 0.0942 \\
\hline ETA0 & 2 & 0.4039 & 0.4037 & 0.4034 & 0.9069 & $\mathrm{MC}$ & -0.1995 & 0.5575 \\
\hline
\end{tabular}

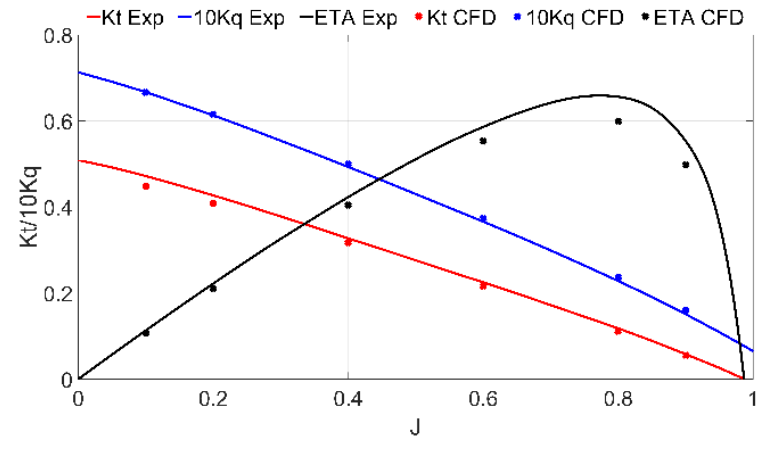

Fig. 3. Comparison between Experimental data (Exp) and CFD Results

TABLE V. Summary of validation results

\begin{tabular}{|c|c|c|c|c|c|}
\hline Variables & $\mathbf{U}_{\mathbf{S N}} \mathbf{( \% )}$ & $\mathbf{U}_{\mathbf{D}}(\mathbf{\%})$ & $\mathbf{U}_{\mathbf{V}}(\mathbf{\%})$ & $\mathbf{E}(\mathbf{\%})$ & Status \\
\hline $\mathbf{K t}$ & 0.2016 & 5.00 & 5.004 & 2.902 & Validated \\
\hline $\mathbf{1 0 K q}$ & 0.0092 & 5.00 & 5.084 & 1.569 & Validated \\
\hline ETA0 & 0.0113 & 10.00 & 10.064 & 4.546 & Validated \\
\hline
\end{tabular}

The results illustrated in Fig. 3 show good agreement with the experimental open-water curve data. However, the peak of the efficiency curve was under-predicted. The efficiency of a propeller is evaluated using thrust and torque coefficient as illustrated in (6). Hence, the error in both the thrust and torque coefficient at high advance coefficient were carried forward when tabulating the efficiencies which resulted in under prediction. However, as illustrated in Table $\mathrm{V}$, the percentage error for all three variables are less than 5\%, which is acceptable. Hence, this proved that the applied methodology could be used to predict the open-water curve for the Wageningen B-Series twin-bladed propeller.

\section{Systematic Simulations}

\section{A. Open-water Curve}

The open-water curve for the twin bladed Wageningen B-Series used by the Explorer AUV was predicted through CFD method. The methodology used for the prediction is similar to the methodology employed previously when determining the openwater curve of the five-bladed propeller. The open-water curve for the twin bladed Wageningen B-Series propeller is as illustrated in Fig. 4. The predicted open-water propeller curve will be used to program all subsequent virtual disc simulations in this study.

\section{B. Virtual Disc}

As mentioned earlier, virtual disc solves the body forces generated as an equally distributed force over a cylindrical disc while ignoring the true geometry of the propeller. This approach greatly reduces the computational solving time. Hence, this approach was used to perform five different rotational speeds and its computational results are compared against the openwater trials conducted by ISE in March 2017. The rotational speeds used are listed in Table VI.

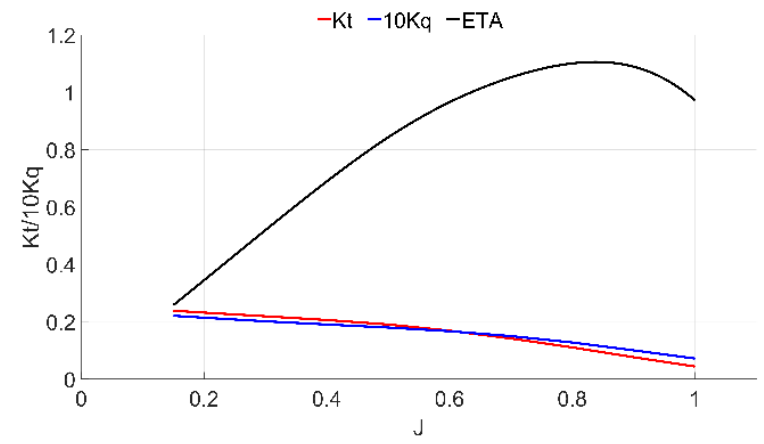

Fig. 4. Twin bladed Wageningen B series open-water curve. 
TABLE VI. Rotational speed used for simulations

\begin{tabular}{|c|c|}
\hline Runs & Rotational Speed, n (RPS) \\
\hline 1 & 2.118 \\
\hline 2 & 2.06 \\
\hline 3 & 2.6 \\
\hline 4 & 3.106 \\
\hline 5 & 3.833 \\
\hline
\end{tabular}

These rotational speeds were used during the open-water trials. Furthermore, the selected rotational speed ensured the advance ratio remain constant at approximately 0.74 . The main interest of this paper is the period when the AUV travels in a straight line, thus the experimental data were analysed to retrieve the period when the AUV was travelling in a straight line. In additional, the experimental data was processed to remove any environmental forces experienced by the AUV such as underwater current.

\section{Direct Simulations}

Direct simulations involve modelling the twin bladed propeller of the AUV as it is, that is considering all its features and geometries. Although this method requires longer computational time, it should offer a higher accuracy solution for the investigating problem. Due to the long computational time, only 1 rotational speed (3.106 RPS) was performed and presented in this paper. Likewise, simulation was performed only for when the AUV is travelling in a straight-line with no hydroplanes attached.

\section{RESULTS AND DISCUSSION}

The results obtained from the simulations are listed in Table VII. As illustrated from the results, the recorded velocity and advance ratio from simulations without hydroplanes are higher than that from the experimental data, resulting in percentage errors ranging from $23 \%-33 \%$. This high percentage error is unacceptable for any CFD simulation.

However, this phenomenon is as expected due to the exclusion of hydroplanes which would significantly change the overall dynamics of the vehicle. The lack of hydroplanes would reduce the overall drag experienced by the AUV hence causing the hydroplanes to travel at a higher speed as compared to experimental data.
However, from the results, it was also observed that there is an anomaly at 3.106 RPS. The percentage error between Star$\mathrm{CCM}+$ result and experimental result is approximately $5 \%$. Hence this rotational speed was selected to be further investigated using the direct simulation method.

The percentage error obtained is higher than the percentage error obtained through virtual disc simulation of $5 \%$. This higher percentage error is viewed as an accurate representation of the investigated problem due to the lack of hydroplanes present in the CFD model as discussed earlier.

\section{CONCLUSION}

Two different methods of representing a propeller within CFD are presented in this paper, 1) virtual disc and 2) discretized propeller. Virtual disc method ignores the geometry of the propeller of interest and solves the body force generated equally over a cylindrical disc. Direct simulation considers all features of a propeller and utilizes the built-in overset mesh function in CFD software to solve the body force generated. Although, the latter method would require a longer computational time, this method would prove to offer higher accuracy solutions when compared to the virtual disc method.

With direct simulation, the lack of public available openwater curve of the Wageningen B-Series propeller was evaluated. The predicted open-water curve was used for all subsequent virtual disc simulations.

A series of rotational speeds was selected from the openwater trial conducted by ISE in March 2017. These rotational speeds were selected after analysing the experimental data to obtain the period when the AUV is travelling in a straight line. This was done to reduce computational time and focus solely on the modelling of propeller for the AUV. The simulated results from the virtual disc method has shown that the percentage error is higher than $20 \%$. However, since all simulations have excluded the effects of hydroplanes, this high percentage error is acceptable and expected. There was an anomaly at 3.106 RPS and hence this rotational speed was selected for the direct simulation. The percentage error obtained is higher than the percentage error obtained from virtual disc simulation of $5 \%$. The higher percentage error obtained will be viewed as an accurate presentation of the investigated problem due to the lack of hydroplanes present in the CFD model.

TABLE VII. Comparison between Experimental data and Star-CCM+ results for simulations using virtual disc

\begin{tabular}{|c|c|c|c|c|c|c|}
\hline Runs & $\mathbf{n}(\mathbf{R P S})$ & J_Exp & Va_Exp (m/s) & J_Star-CCM+ & Va_Star-CCM+ (m/s) & Error (\%) \\
\hline 1 & 2.06 & 0.748 & 1.016 & 0.938 & 1.256 & 1.291 \\
\hline 2 & 2.118 & 0.739 & 1.03 & 0.938 & 1.592 & 23.62 \\
\hline 3 & 2.6 & 0.746 & 1.203 & 0.942 & 1.900 & 32.33 \\
\hline 4 & 3.106 & 0.732 & 1.806 & 0.941 & 2.349 & 5.193 \\
\hline 5 & 3.833 & 0.748 & 1.866 & 0.943 & & 25.91 \\
\hline
\end{tabular}




\section{VIII.FUTURE WORK}

The current research will proceed to complete the direct simulations. Upon completion, the current research will perform free-running simulations of the AUV through various zig-zag manoeuvres and turning circles manoeuvres cases. In all subsequent simulations, hydroplanes will be included and the hydrodynamic coefficients of the AUV will be evaluated and benchmarked against analytical method performed by ISE.

\section{REFERENCES}

[1] A. Phillips, S. Turnock, and M. Furlong, "The use of computational fluid dynamics to aid cost-effective hydrodynamic design of autonomous underwater vehicles," Proceedings of the Institution of Mechanical Engineers, Part M: Journal of Engineering for the Maritime Environment, vol. 224, no. 4, pp. 239-254, 2010.

[2] W. J. M. Rankine, "On the mechanical principles of the action of propellers," Transactions of the Institution of Naval Architects, vol. 6, 1865.

[3] R. Broglia, G. Dubbioso, D. Durante, and A. Di Mascio, "Simulation of turning circle by CFD: Analysis of different propeller models and their effect on manoeuvring prediction," Applied Ocean Research, vol. 39, pp. 1-10, 2013.

[4] V. I. Krasilnikov, "Self-propulsion RANS computations with a single-screw container ship," in 3rd International Symposium on Marine Propulsors smp, 2013, vol. 13.

[5] C. Delen, S. Sezen, and S. Bal, "Computational investigation of self propulsion performance of
DARPA Suboff vehicle," TAMAP J. Eng., pp. 1-12, 2017.

[6] A. Mofidi and P. M. Carrica, "Simulations of zigzag maneuvers for a container ship with direct moving rudder and propeller," Computers \& Fluids, vol. 96, pp. 191-203, 2014.

[7] N. Zhang and S. L. Zhang, "Numerical simulation of hull/propeller interaction of submarine in submergence and near surface conditions," Journal of hydrodynamics, ser. b, vol. 26, no. 1, pp. 50-56, 2014.

[8] J. E. Martin, T. Michael, and P. M. Carrica, "Submarine maneuvers using direct overset simulation of appendages and propeller and coupled CFD/potential flow propeller solver," Journal of Ship Research, vol. 59, no. 1, pp. 31-48, 2015.

[9] Y. Jin, J. Duffy, S. Chai, C. Chin, and N. Bose, "URANS study of scale effects on hydrodynamic manoeuvring coefficients of KVLCC2," Ocean Engineering, vol. 118, pp. 93-106, 2016/05/15/ 2016.

[10] F. Stern, R. V. Wilson, H. W. Coleman, and E. G. Paterson, "Verification and validation of CFD simulations," IOWA INST OF HYDRAULIC RESEARCH IOWA CITY1999.

[11] O. E. Moctar, V. Shigunov, and T. Zorn, "Duisburg Test Case: Post-panamax container ship for benchmarking," Ship Technology Research, vol. 59, no. 3, pp. 50-64, 2012. 